\title{
Levels of Physical Activity Participation of the Staff of Universiti Selangor.
}

\author{
${ }^{1}$ Mohd Norazwan A. Bakar, ${ }^{2}$ Nasrudin Md. Rahim \\ ${ }^{I}$ Faculty of Education/Unversity Selangor, Malaysia) \\ ${ }^{2}$ (Faculty of Science and Mathematic/ University Selangor, Malysia)
}

\begin{abstract}
This study is carried out to identify the levels of the Universiti Selangor (Unisel) staff's participation in physical activities. It aims to investigate the stages of their physical activities to determine whether these activities are beneficial to them or otherwise. This study is a descriptive survey research of which the data has been collected via questionnaire from 231 employees of Unisel in Bestari Jaya Campus. Respondents are asked questions about their physical activities based on the International Physical Activity Questionnaire (IPIQ). The findings reveal that many members of the staff of the Universiti Selangor practice physical activities as their way of life. However, the level of their participation in physical activities has not reached a satisfactory level. The respondents often carry out physical activities, but mostly their participation is still considered within the range of average to low level. The respondents' participation in physical activities is influenced by their attitude which seeks acknowledgment rather than practicing it as a healthy lifestyle. The findings shows that the employees' physical activity participation is very high. However, they do not carry out the activities according to the principles of physical activity domain. This study concludes that there are still many Unisel staff members that seldom treat physical activities as their everyday routines. The findings show that 106 of the respondents carry out physical activities at a low level, 49 of the respondents at the medium level and 76 of the respondents at a high level. The findings are insufficient to establish that the respondents have carried out the best physical activity practice. The results conclude that these Unisel staff members still need to be guided and educated in order to ensure that their participation in physical activities becomes a healthy lifestyle that is led by the whole community.
\end{abstract}

Keywords: Employee, Low, Medium and High Level, Physical Activities

\section{Introduction}

Today, we live in an era in which our industrialized society is progressive and continuously active. The atmosphere in these modern times is much different from that of yesteryear's agricultural community. In modern society, people are constantly busy and working against the clock. People quickly become fatigued, stressed out, and often lack a sense of tranquility. As a result, many people feel that they do not have enough free time to take up challenging activities outside of their normal daily undertakings. The pattern of modern life, coupled with the advancement of science and technology has made the human race lethargic. Humans no longer need to use physical energy to work. Most work is done with the aid of computers and sophisticated machines and tools. This lack of physical activity and exercise can inevitably be linked with many chronic diseases such as cardiovascular disease, diabetes, and obesity (Braith and Stewart, 2006)

Nevertheless, there are a variety of physical exercises and activities that can be completed according to individual interests and compatibility. Physical exercises can contribute to improving one's general health and overall fitness level in particular. These fitness components include cardiac endurance, muscle strength, body composition, flexibility, body balance, speed, and power (Anspaugh, Hamrick, and Rosato, 2003).

Most people are aware that involvement in regular physical activities can provide lasting benefits, however, public participation in physical activity is often disregarded.. Among the people who often avoid physical activities are blue collar workers who work indoors. Incuded among these are the staffs of higher education institutions. This phenomenon is worrisome as the number of employees in these various institutions increase from time to time. It is alarming that if this situation continues, there is a possibility that this will affect their work performance.

Many people have the wrong impression about physical activity. The question is: how we can identify the physical activities which are most beneficial to the body? Every employee should understand the varying extent of benefits from these activities so as not to feel too quickly satisfied with what he/she practices. For example, an employee who jogs for less than ten minutes regularly feels that he has already performed a healthy physical activity.

It has been reported that regular and periodic physical activities are associated with a reduced incidence of cardiovascular disease and mortality (Engstrom, 1999). A dynamic exercise, such as running, will increase cardiac output as a result of increased heart rate and cardiac stroke volume. Blood supply will be directed to the 
muscles which are working rather than the non-working muscles and visceral organs. This in turn will increase the systolic blood pressure but will not cause significant changes in the diastolic blood pressure. It can also decrease vascular resistance. Isometric training is also known to increase systolic and diastolic blood pressure at which the magnitude depends on the size of the muscles involved as well as the burden imposed upon the muscles (Pollock and Schmidt, 1995).

Physical fitness is an important aspect of our lives. People who lead a healthy lifestyle are more productive and more confident when facing challenges in life. Higher Learning Institution staffs can be considered a model for others because they have a conducive environment in terms of facilities. Well-organized and systematic universities provide ample space for employees to increase their knowledge and to obtain vast knowledge. In reality, there is no difference in the perceptions towards the physical activities performed. What makes them different is that when choosing a physical activity to perform, the choice must be based on the correct knowledge. Some staff members prefer jogging and brisk walking as their physical activities, while others prefer some other type of outdoor physical activitie. Thus, an employee must know which physical activity is best suited for him/her so that the activity will produce a positive impact towards a healthier lifestyle.

\section{Research Objectives}

This study intends to achieve a number of objectives. The first objective is to identify the types of physical activities that are performed by the staff of a university in Malaysia, and whether those activities are considered heavy, moderate, or light. The second objective is to identify the categories of university staff who regularly perform physical activities, eventhough their participation in physical activities has yet to be considered to have a significant overall impact

\section{Problem Statement}

The problem being investigated is as to why the staff of the University Selangor finds it so difficult to take the time for physical activities such as exercising or playing sports. There are some employees who participate in physical activities, however, from the context of fitness, their participation is not considered adequate. Despite the fact that the sports facilities at the university are sufficient, there are still many who do not take part in physical activities. This statement is based on the analysis of the record of attendance of participation in the log book of sports facilities at the University Selangor.

Based on this problem statement, a study of the level of physical activity participation among the University Selangor staff needs to be conducted. This study was conducted based on the awareness that the understanding of physical activities is crucial in helping increase the staff's knowledge of healthy lifestyles. According to Noor Aini (2004), knowledge of dietary and physical exercise habits influences the everyday lifestyles of civil servants. This is of the utmost important because a healthy lifestyle practiced by employees has an impact on the quality of work produced by the staff, and in turn impacts the performance of the department.

\section{Significance Of The Study}

This study is important because not much research has been carried out on this matter in the past. Such research is very welcomed because any findings will help the staff to practice physical activities or muscle movement more effectively. The lack of specific research to evaluate the effectiveness of campaigns to promote physical activities as well as a growing number of patients with cardiovascular deseases proves that the effectiveness of such campaigns can be questioned (Ministry of Health Malaysia, 2011).

Realizing this, the present researcher was inspired to carry out a specific research related to the physical activities undertaken by university staff members aimed at creating a healthier lifestyle. The results of this research will be beneficial to many individuals and groups. Among the individuals who could benefit from this research are people who do not exercise very much. In addition, teenagers, adults, middle-aged women, and the elderly can also benefit from this research. It is hoped that the findings of this study will have an significant impact on these groups of people in moving towards a healthier lifestyle.

\section{Research Instrument}

The instrument used to collect the data for this research was a questionnaire. The questionnaire was adapted from a questionnaire that had been used in previous studies. This questionnaire was adapted from the International Physical Activity questionnaire (IPAQ) which was used in a study in November 2005 in Canada. Section A of the questionnaire contains questions related to the respondents personal details such as gender, age, race, weight, and height. Section B consists of seven questions. All of these questions required the respondents to think of any physical activities that they had done in the past seven days. First, the question asked about the number of days the respondents had performed these physical activities. Examples of these physical activies included lifting heavy things, shovelling, aerobics workout, playing sports or speed cycling. The second 
question asked about the amount of time spent on these activities. The third question asked about the number of days the respondents performed moderate physical activities. Some examples of moderate physical activities include lifting light things, mopping the floor, cycling, washing a car, or playing badminton. The fourth question asked about the number of hours spent on these activities. The fifth question asked whether the respondents walk at work or do any other recreational activities. The sixth question asked about the number of hours taken by the respondents to perform these activities within a day. The seventh question asks about the amount of time spent by the respondents resting or sitting at work, including the time spent reading or lying down to watch television.

VI. Findings

\begin{tabular}{l|l|c|c|c}
\hline \hline NO & \multicolumn{1}{|c|}{ ACTIVITIES } & FREQUENCY & PERCENTAGE & TOTAL PERCENTAGE \\
\hline 1 & $\begin{array}{l}\text { low - not categorized as moderate } \\
\text { or high }\end{array}$ & 106 & 45.9 & 45.9 \\
\hline 2 & $\begin{array}{l}\text { Moderate - minimum of 600MET } \\
\text { minute/per week }\end{array}$ & 49 & 21.2 & 21.2 \\
\hline 3 & $\begin{array}{l}\text { high - overall total of at least 3000 } \\
\text { MET minute/per week }\end{array}$ & 76 & 32.9 & 32.9 \\
\hline & TOTAL & 231 & 100.0 & 100.0 \\
\hline \hline
\end{tabular}

\section{Conclusion}

The findings show that the level of physical activity of the University of Selangor Staff members as practiced by 106 respondents was relatively low when compared to the 49 respondents who performed at a moderate level of physical activities, and the 76 respondents who performed at a high level of physical activities. Even though there are opinions which suggest that if the respondent had not done the lower level physical activities, they would have done the high level physical activities. However, the researcher questions as to why the respondents felt that they had actually done physical activities when in reality these activities had not provided any positive effects, especially on the cardiovascular system. The implications of these findings led the researcher to look deeper into the University of Selangor's staff members interpretation concerning proper exercises. Respondents who performed low level physical activities often felt that they had, all along, been practicing suitable physical activities as part of their lifestyle. Respondents who had participated in moderate level physical activities also needed some attention. There was the possibility that these respondents were more inclined not to do any physical activities at all. This statement is based on the observations of the researcher as to the actual level of awareness on health and fitness issues possessed by the staff members of the University of Selangor.

\section{References}

[1]. Anspaugh, D. J, Hamrick, M. H dan Rosato, F. D 2003. Wellness Concept and Applications. Ed ke 5. New York: McGraw.

[2]. Braith, R. W and Stewart, K. J (2006). Resistance Exercise Training: It's Role in the Prevention of Cardiovascular Dieses. Journal of the American Hearth Assiciation. 113:2642-2650.

[3]. Engstrom, G. (1999). Hypertensive Men Who Exercise Regularly Have Lower of Cardiovascular Mortality. Journal of Hypertension 1999, 17:737-742.

[4]. Noor Aini, A. H., Goon, J. A., Aminuddin, A. H. K., Azian, A. L., Nabishah, M., Zaiton, Z., Jamilah, B., Roshidah, H., Musalmah, M. and Wan Zurina, W. N. (2004). Effect of Exercise (Tai Chi) on Oxidative Status Among Population in Retirement Age: A Preliminary Report. Malaysian Journal of Biochemistry and Molecular Biology. 9, 31-34.

[5]. Pollock, M. L dan Schmidt, D. H. (1995). Heart Disease and Rehabilitation. (2nd edit). Human Kinetics.345-347. 